\title{
ESSAI D'UNE TABLE NON-VIBRANTE PAR ENREGISTREMENT DU MOUVEMENT BROWNIEN D'UN GALVANOMETRE
}

\author{
par P. B. ROTTIER ET M. T. JANSEN \\ Laboratorium voor Histologie en Mikroskopische Anatomie der Universiteit Utrecht, \\ Nederland
}

\section{Synopsis}

Attempts to attain the natural limit of the sensitivity of a galvanometer, viz. the $\mathrm{Br}$ ow $\mathrm{n}$ i a movement, have at first been unsuccesful in our institute. Neither a heavy slab of stone attached to an outer wall, nor a masonry of $1800 \mathrm{~kg}$ in the cellar embedded in a layer of sand $10 \mathrm{~cm}$ thick gave our thermorelais enough stability against the vibrations always present in buildings.

Only by putting the thermorelais on a base with an own frequency much lower than that for which galvanometers are particularly sensitive (some 15 periods per second) we could surmount the difficulties. Such a base, supported by and oscillating freely on four rubber blocs with an oscillating frequency of about 2 periods per second, has been built and described. It has been necessary to lessen its free movements by placing pieces of rubber under the cricks meant for the construction of the whole.

On this table with a weight of $2600 \mathrm{~kg}$ the $\mathrm{B} \mathrm{r}$ o w n i a n movement of a galvanometer has been recorded without any interference of the vibrations of the building.

Introduction. O r n s te i n, $\mathrm{M}$ oll et $\mathrm{B}$ u r g e r ont décrit à la page 18 de leur traité "Objektive Spektralphotometrie" 1 ) la façon d'agrandir par moyen d'un thermorelais ${ }^{2}$ ) l'amplitude d'un galvanomètre jusqu'à ce que tout agrandissement supplémentaire n'eût plus de sens, le mouvement Brownien de ce galvanomètre devenant visible. L'écart moyen de ce mouvement en volt se calcule par:

$$
\overline{B r}=1,13 \cdot 10^{-10} \sqrt{R / t}
$$

où $R$ est la résistance en $\mathrm{Ohm}$ du circuit électrique du galvanomètre primaire et $t$ le temps d'indication en secondes du thermorelais entier.

D'abord nous n'avons pas réussi à atteindre cette limite naturelle 
de la sensibilité d'un galvanomètre dans notre institut, qui se trouve près des terrains fréquentés de la gare et qui est bâti sur un sol mou. Un plateau de pierre de $90 \times 40 \times 6 \mathrm{~cm}$, fixé dans un mur extérieur au premier étage, n'offrit pas assez de stabilité. Il va sans dire que la mesure obtenue dans le balancement de la bobine du galvanomètre primaire joue un grand rôle dans l'insensibilité du galvanomètre à l'égard des vibrations des bâtiments, mais nous n'avons pas pu obtenir un galvanomètre mieux balancé. Le transport du thermorelais à la cave où il fut placé sur une construction en pierres briques, longue de 4 mètres, haute de $70 \mathrm{~cm}$, large de $50 \mathrm{~cm}$, couverte d'un plateau de pierre épais de $6 \mathrm{~cm}$, le tout pesant $1800 \mathrm{~kg}$ et reposant sur une couche de sable de $10 \mathrm{~cm}$, ne donna aucune amélioration. Au contraire, la situation était pire qu'au premier étage.

Une analyse du problème, où les pùblications de $\mathrm{H} \mathrm{a} \mathrm{r}$ i $\mathrm{n} \mathrm{g} \mathrm{x}^{3}$ ) nous ont guidés, nous a montré qu'il ne s'agit pas en premier lieu d'éviter les vibrations de l'entourage, maịs d'obtenir l'accord le plus petit possible entre la fréquence dominante de l'entourage et celle pour laquelle le galvanomètre est le plus sensible.

Les galvanomètres de $\mathrm{M}$ oll, que nous avons employés, semblent être le plus sensibles pour des vibrations d'une fréquence approximative de 15 périodes par seconde, tandis que la fréquence des vibrations des bâtiments est précisément de cet ordre de grandeur. Or, une couche de sable de $10 \mathrm{~cm}$, comme c'était le cas dans notre disposition dans la cave, amortit très bien les vibrations d'une fréquence élevée - 100 périodes par seconde et plus - mais elle n'a presque aucune influence sur les vibrations de 15 périodes par seconde. Par contre le plateau que nous avons employé au premier étage ne peut pas vibrer avec une fréquence de petit nombre - environ 15 - mais il vibre plutôt avec une fréquence élevée. Cette différence entre le nombre de vibrations des deux dispositions que nous avons essayées est la cause, que nos résultats ont été meilleurs sur le plateau de pierre au premier étage que sur la maçonnerie dans la cave.

Mais puisque les deux dispositions ont été insuffisantes pour nous faire atteindre notre but - l'enregistrement du mouvement Brownien - nous avons dû chercher pour notre thermorelais une base avec une fréquence propre de vibration, qui s'écartait le plus possible de celle de 15 périodes par seconde, pour laquelle les galvanomètres sont sensibles. En plus cet écart doit être cherché du côté des petits nombres plutôt que du côté des nombres élevés pour obtenir la 
moindre transmission des vibrations perturbatrices de l'entourage.

Or, le groupement ,, Recherches des tensions et des vibrations" du TNO à Delft nous a fait les calculations pour une construction avec une fréquence propre de vibrations d'à peu près 2 périodes par seconde (voir le rapport No. VII, de Ir. R. G. B o i t e n). La construction devant servir à des travaux optiques, nous avons indiqué auparavant les dimensions de $4 \times 0,6 \times 0,7$ mètres. Elle a été bâtie dans la cave de notre institut.
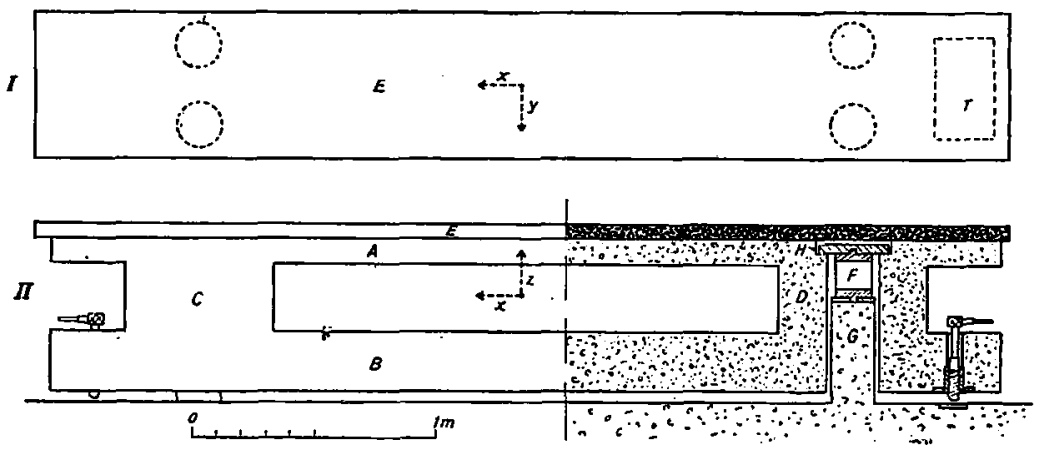

Fig. 1. La table non-vibrante.

I. Vue du haut.

II. Vue du côté.

Voir le texte pour l'explication des caractères.

Description de la table non-vibrante. La figure 1 représente la table en béton armé, vue du haut et vue du côté. La vue du côté montre des ouvertures aux deux bouts et au milieu pour avoir une meilleure répartition de la masse. Ces ouvertures divisent le bloc en deux parties $A$ et $B$, qui sont attachées par les colonnes $C$ et $D$ rectangulaires de profil. Il se trouve un plateau de pierre dure $\mathrm{E}$ dessus.

Le poids de toute la construction - à peu près $2600 \mathrm{~kg}$ - est supporté par 4 cylindres de caoutchouc vulcanisés entre deux tôles d'acier, munies de cames. Ces cylindres se trouvent dans les colonnes $\mathrm{C}$ et $\mathrm{D}$ et leur projection est indiquée en pointillage dans la figure 1 , la vue du haut. La moitié droite de la vue du côté, représentée en coupe, montre bien le support de la construction. Le cylindre en caoutchouc $F$ se repose avec sa tôle de dessous sur la colonne en béton armé $G$, qui est placée sur le sol. La tôle de dessus du cylindre F s'attache avec sa came à une tôle d'acier $\mathrm{H}$, qui est fixée par huit barres d'attache à un anneau maçonné solidement dans le béton de la con- 
struction. Par cet anneau, les barres d'attache et la tôle d'acier $\mathrm{H}$ le poids.de la construction est transporté au cylindre de caoutchouc.

On a accès aux blocs de caoutchouc en enlevant le plateau $E$ et en détachant ces tôles d'acier. Dans ce cas le poids de la construction est pris par trois crics, qui ont été maçonnés dans le béton aux deux bouts de la construction, deux à droite, un à gauche. En outre la construction, qui subit facilement des oscillations de quelques centimètres quand on la pousse, peut être immobilisée par ces crics.

Les cylindres de caoutchouc s'enfoncent plus ou moins quand la table se meut de haut en bas ou bien tourne autour des axes $x$ et $y$, indiqués dans la figure 1. Quand elle oscille dans la direction des axes $x$ et $y$ et quand elle tourne autour de l'axe $z$, les cylindres tendent à glisser entre les deux tôles. C'est la raison que celles-ci ont été vulcanisées au caoutchouc des cylindres et fixées par leur cames à la face supérieure des colonnes comme à la tôle d'acier $H$ de la table.

En projetant l'appareil on s'est efforcé. à faire coïncider le centre élastique du système des ressorts avec le centre de gravitation de la table. Dans ce cas une façon d'osciller n'entraînera pas l'autre, de sorte que par exemple un mouvement causé par des oscillations transversales ne changera pas la position horizontale de la face supérieure.

La construction possède les fréquences propres suivantes: translations dans la direction de l'axe $x: 1,3$ périodes/seconde.

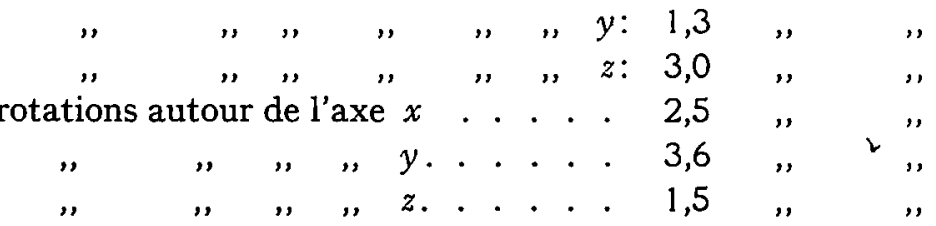

Les cylindres de caoutchouc ont été vulcanisés de telle façon qu'ils garantissent en outre un bon amortissement de la table. Pour les oscillations transversales dans la direction de l'axe $x$ nous avons déterminé un décrement logarithmique du système des ressorts de 0,04 comme mesure des qualités d'amortissement. C.v.d. que l'amplitude de l'oscillation amortie est réduite de moitié en 6 secondes.

Enregistrement des amplitudes du thermorelais. a) L e s $\mathrm{m}$ é $\mathrm{t} \mathrm{h}$ ode s:

Le thermorelais (de la S.A. Kipp, Delft) fut placé sur la table décrite ci-dessus tout à droite comme c'est indiqué par $T$ dans la figure 1. Le relais a un temps d'indication de \pm 2 secondes. Le galvanomètre primaire (type original de Moll, résistance interne de $55 \mathrm{Ohm}$, nommé ici le A 1) a un 
temps d'indication apériodique de $\pm 1,5$ secondes. Comme galvanomètre secondaire nous avons employé le microgalvanomètre de $M$ o 11 avec un temps d'indication de 0,2 secondes (nommé ici le A 23) et le galvanomètre à bobine double (type $\mathrm{Kc}$, nommé ici le A 54) avec un temps d'indication apériodique de 12 secondes à peu près. La première combinaison, A 1-A 23, a un temps d'indication de 3,5 secondes, la seconde, A $1-\mathrm{A} 54$, de \pm 15 secondes. L'amplitude du galvanomètre secondaire a été enregistrée par voie photographique. La distance du papier sensible au miroir du galvanomètre était de 1,2 mètres. Le galyanomètre primaire était shunté d'une résistance extérieure de $10 \mathrm{Ohm}$ par un banc réducteur avec lequel il était possible d'appliquer de petites tensions de valeur connues pour calibrer le thermorelais.

Pour savoir la déviation moyenne des amplitudes une droite fut tracée par ou au long des enregistrements. Chaque $\mathrm{mm}$ la distance de l'enregistrement à cette droite dans le sens de l'axe du tambour d'enregistrement fut mesurée à $0,1 \mathrm{~mm}$ près. L'addition fut faite de toutes les valeurs, positives ou négatives, le total des deux divisé par leur nombre respectif, puis le moyen des deux quotients tiré pour établir la position de la ligne zéro. Puis l'addition fut faite des carrés des distances des valeurs mesurées de la ligne zéro calculée et le total fut divisé par le nombre des valeurs. Enfin la racine de ce quotient donne la déviation moyenne cherchée de l'enregistrement.

b) Mouvement Brownien enregistré sur la $\mathrm{table} n$ on-vibrante entièrement libre. Les figures 2 et 3 représentent des dessins, réduits à moitié, de quelques enregistrements faits avec le thermorelais placé sur la table nonvibrante. Les lignes $a$ et $b$ ont été prises sur la table entièrement libre avec la combinaison A 1-A 54, la première avec une vitesse d'enregistrement de 43,1 secondes pour $10 \mathrm{~mm}$, la deuxième avec 5,5 secondes pour $10 \mathrm{~mm}$. La déviation moyenne des deux lignes a été après mesure $1,1 \mathrm{~mm}$. Des tensions de $1,7 \cdot 10^{-9} \mathrm{~V}$ mises au galvanomètre primaire ont donné une amplitude moyenne de $8 \mathrm{~mm}$. Calculé avec la formule d'en haut la déviation moyenne du mouvement Brownien de l'ensemble employé fut:

$$
\overline{B r}=1,13 \cdot 10^{-10} \sqrt{\frac{65}{15}}=2,35 \cdot 10^{-10} \mathrm{~V}
$$

ce qui correspond à $1,1 \mathrm{~mm}$.

L'accord des deux valeurs étant parfait, il est évident que les vibrations du dehors n'étaient plus visibles dans ces enregistrements, qui ne démontrent que le mouvement Brownien du galvanomètre primaire.

L'enregistrement $c$ de la figure 3 a été fait avec la combinaison 
A 1-A 23. Une tension de $1,07 \cdot 10^{-7} \mathrm{~V}$ a donné une amplitude de 34,5 $\mathrm{mm}$. La vitesse d'enregistrement a été 5,5 secondes pour $10 \mathrm{~mm}$. La déviation moyenne de la ligne a été mesurée sur un agrandissement photographique $(4,57$ fois $)$. Elle était $0,13 \mathrm{~mm}$.

Le $\overline{B r}$ calculé $=1,13 \cdot 10^{-10} \sqrt{\frac{65}{3,5}}=4,87 \cdot 10^{-10} \mathrm{~V}=0,16 \mathrm{~mm}$.

L'accord n'est pas si parfait qu'auparavant ce qui s'explique par la petitesse des déviations mesurées. Tout de même on peut conclure que le mouvement Brownien du galvanomètre primaire seul a été enregistré et que les vibrations perturbatrices de l'entourage n'étaient pas perceptibles.

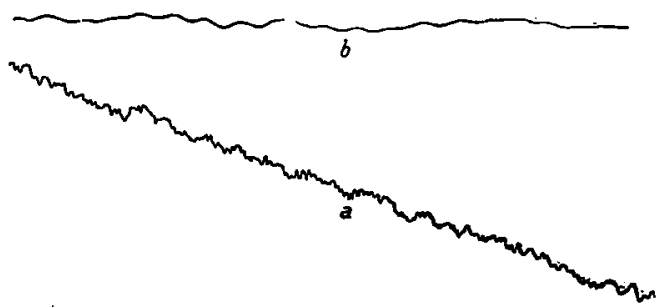

Fig. 2. Des enregistrements de l'amplitude du galvanomètre secondairc d'un thermorelais placé sur la table non-vibrante. Réduit à moitié. Combinaison A 1-A 54, deux vitesses d'enregistrement. Le mouvement B r o w n i e n pur. Voir le texte.

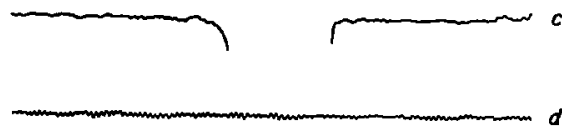

Fig. 3. Des enregistrements de l'amplitude du galvanomètre secondaire d'un thermorelais placé sur la table non-vibrante. Réduit à moitié. Combinaison A 1-A 23. Les lignes $c$ et $e$ ne démontrent que le mouvement $\mathrm{Br}$ ow nie n pur. La ligne $d$ est très contaminée par des vibrations du bâtiment, la ligne $f$ est peu contaminée. Voir le texte.

c) Mouvement Brownien mesuré surla table $\mathrm{pas} t$ o u t à $\mathrm{fa}$ it $\mathrm{libr}$. De ce qui précède il résulte que la construction décrite ici remplissait entièrement son but. Nous 
avons réussi à enregistrer sur elle le mouvement électrique et inévitable du circuit du galvanomètre primaire sans qu'aucune vibration de l'entourage y fût dedans. Ceci a été fait dans notre institut qui n'est pas très bien situé à l'égard des vibrations et avec un galvanomètre primaire comme on les achète sans qu'il ait été spécialement balancé.

Mais la suspension de la construction sur des blocs de caoutchouc la rend tellement mobile, que d'autres travaux y sont difficiles à faire. En plus la faculté du caoutchouc d'être enfoncé donne des changements de position de la face supérieure de la table quand on y met ou déplace des objets. Un galvanomètre dont la bobine n'est pas parfaitement balancée - ce qui est très fréquent - réagit sur des changements de position. C'est ce que nous avons remarqué avec nos enregistrements. Un poids de 10 grammes mis au bord du plateau supérieur suffisait à faire basculer la construction lourde de $2600 \mathrm{~kg}$ de telle façon pour que le thermorelais produisit des amplitudes reproductibles de quelques dizaines de $\mathrm{mm}$. Et quand une personne se déplaçait pendant un enregistrement d'une distance de quelques mètres jusqu'au côté de la table, le sol de béton de la cave céda suffisamment pour faire basculer la construction. Un déplacement de l'enregistrement sur le papier sensible en était la suite.

Puisque la table devait être employée pour plusieurs travaux optiques qui ne demandaient pas toujours la sensibilité maximum du thermorelais, nous avons cherché à l'immobiliser sans trop introduire des perturbations de l'entourage. Nous y avons réussi en mettant des morceaux de caoutchouc dur de $5 \times 5 \times 2 \mathrm{~cm}$ sous les trois crics, avec lesquels la construction peut être élevée. Les crics ont été tournés un peu jusqu'à ce que chacun d'eux portât quelques $\mathrm{kg}$. Les vibrations du sol introduites ainsi sont montrées par l'enregistrement $d$ de la figure 3. Les dates de $d$ diffèrent de celles de $c$ en tant que chez $d$ l'échelle de l'amplitude du thermorelais a été plus petite de $9 \%$. Comparé à $c$ la ligne $d$ montré bien que les amplitudes irrégulières ont été plus grandes et démontrent des battements typiques qui sont causés par l'interférence des oscillations diverses de la table.

Le gain indubitable en stabilité par la mise des morceaux de caoutchouc sous les crics a donc détérioré les qualités non-vibrantes de la table de telle façon, que nous avons cherché à améliorer le mauvais balancement de la bobine du galvanomètre primaire. Le professeur H. C. Burger nous y a indiqué la voie. Un peu de laque en solu- 
tion alcoolique a été mis sur un des côtés de la bobine. Pour savoir sur quel côté et la quantité à appliquer, le galvanomètre a été basculé en avant et en arrière en faisant glisser un prisme de ver plat sous l'appui du derrière du galvanomètre. En même temps l'image d'une lampe dans le miroir du galvanomètre a été observée avec un télescope.

Le galvanomètre $\mathrm{A} 1$ ainsi mieux balancé a été essayé dans la combinaison A 1-A 23. Il donnait les enregistrements $e$ et $f$ de la figure 3, $e$ avec la table entièrement libre, $f$ avec la table immobilisée par les morceaux de caoutchouc sous les crics. Les autres dates ont été lesmêmes que pour l'enregistrement $c$. La déviation moyenne a été trouvée $0,14 \mathrm{~mm}$ pour $e$ et $0,23 \mathrm{~mm}$ pour $f$, tandis que le mouvement Brownien calculé était toujours $0,16 \mathrm{~mm}$. Comme dans le cas $c$ sur la table libre la valeur calculée était pratiquement d'accord avec la valeur déterminée, tandis que d'après $f$ la table immobilisée par les crics sur des morceaux de caputchouc démontre un mouvement irrégulier un peu plus grand. Mais ce mouvement diffère si peu du mouvement $B$ row $n$ ie $n$ pur que pour des travaux normaux il ne sera pas souvent nécessaire de mettre la table entièrement libre.

La comparaison des enregistrements $f$ et $d$ démontre clairement que le balancement de la bobine du galvanomètre l'a sensiblement amélioré.

Pourtant les faibles vibrations transmises par les crics reposant légèrement sur du caoutchouc se font toujours remarquer dans l'enregistrement $f$. Il est donc invraisemblable qu'on pourra éviter l'emploi d'une table non-vibrante en améliorant uniquement lè balancement de la bobine du galvanomètre.

Résumé. Des essais faits pour arriver à l'enregistrement de la limite naturelle de la sensibilité d'un galvanomètre - le mouvement $\mathrm{B}$ r o w n i e n - ont d'abord échoué dans notre institut. Ni la position du thermorelais sur un plateau lourd dans un mur extérieur, ni la position sur une maçonnerie de $1800 \mathrm{~kg}$, reposant dans la cave sur une couche de sable de $10 \mathrm{~cm}$, ne suffisaient contre l'action des vibrations du bâtiment.

Seule la position du thermorelais sur une construction avec une fréquence propre qui est bien moindre que celle, pour laquelle les galvanomètres sont sensibles (à peu près 15 périodes par seconde) nous peut donner une satisfaction complète: Une construction repo- 
sant et oscillant sur quatres cylindres de caoutchouc avec une fréquence propre d'à peu près 2 périodes par seconde, a été construite et décrite. Il a fallu diminuer sa mobilité assez grande en mettant des morceaux de caoutchouc sous les crics destinés au montage de la construction.

Des enregistrements de l'amplitude d'un thermorelais placé sur la table ont démontré le succès dont les disturbations des vibrations de l'entourage ont été survaincues.

Nous remercions $M$. le professeur $H$. B e rkelbach van der Sprenkel pour la persistance avec laquelle il a poursuivi la réalisation de l'appareil et l'intérêt qu'il a bien voulu nous montrer continuellement.

Reçu 24-2-51.

\section{LITERATURE}

1) Ornstein, L.S., Mo11, W. J. H.et B u rger, H.C., Objektive Spektral-photometrie. Braunschweig, 1932.

2) M oll, W. J. H. et B u r ger, H. C., Phil. Mag. 1 (1925) 624.

3) Haring $x$, J. A., Philips techn. Tijdschr. 9 (1947) 16 et 85. 